\title{
Lentes asféricas: avaliação da indicação clínica e das opções de lentes
}

\section{Aspherical IOLs: clinical evaluation and options}

Newton Kara-Jose Junior ${ }^{1}$, Marcony Rodrigues de Santhiago ${ }^{2}$

\section{Resumo}

Com a utilização de lentes intraoculares (LIOs) asféricas na cirurgia de catarata, o oftalmologista é capaz de oferecer, além da melhora da acuidade visual pela remoção do cristalino opacificado, benefícios adicionais como, por exemplo, a redução de aberrações ópticas oculares, que diminuem a sensibilidade ao contraste.Foram revisadas publicações recentes sobre LIOs asféricas e adicionadas conclusões de nossos estudos na área, a fim de expor neste artigo nossa percepção a respeito da influência da utilização de LIOs que diminuem a aberração esférica do olho, na qualidade visual após a cirurgia da catarata

Descritores: Lentes intraoculares; Catarata; Acuidade visual/fisiologia

\footnotetext{
'Livre-docente, Professor Colaborador da Faculdade de Medicina da Universidade de São Paulo - USP - São Paulo (SP); Chefe do Setor de Catarata do Hospital das Clínicas da Universidade de São Paulo - USP - São Paulo (SP), Brasil;

²Estagiário do Setor de Catarata do Hospital das Clínicas da Universidade de São Paulo - USP - São Paulo (SP), Brasil.
} 


\section{INTRODUÇÃO}

$\mathbf{O}$ olho humano é opticamente imperfei to, assim, a qualidade da imagem projetada na retina é inferior à imagem real do objeto visualizado. Isso ocorre porque ao atravessar estruturas intraoculares, especialmente córnea e cristalino, os raios luminosos sofrem desvios, como dispersão, refração, absorção, além de diversas aberrações. Contudo, a imagem final interpretada no córtex cerebral será de muito melhor qualidade, pois o Sistema Visual tem um mecanismo compensatório (neuroadaptação) para a maioria das deficiências do Sistema Óptico ${ }^{(1-12)}$.

Mesmo considerando a neuroadaptação, acredita-se que a correção de algumas aberrações ópticas pode melhorar ainda mais a qualidade visual. Uma modalidade de imperfeição óptica ocular que não pode ser resolvida com lentes corretoras (óculos), mas é passível de compensação, pela cirurgia de catarata com implante de LIOs especiais, é a aberração esférica (alta ordem), a qual trata-se de uma imprecisão na asfericidade periférica da córnea (a superfície corneal periférica é mais plana do que deveria ser), que prejudica a visão de longe em situações de pouca luminosidade, quando a midríase pupilar permite que os raios luminosos que incidem na periferia corneal sigam em direção à retina; esses raios serão focalizados anteriormente à mácula, diminuindo a qualidade visual ${ }^{(1-5)}$.

Foi demonstrado que, com a idade, a qualidade óptica vai se deteriorando, e uma possível causa desta degradação é a inversão de padrão de aberração esférica do cristalino, de negativo no adulto jovem (que compensaria parcialmente a aberração corneal positiva), para valores negativos menores, ou até positivos quando o cristalino está opacificado ${ }^{(6-12)}$.

As lentes esféricas convencionais são refrativamente similares ao cristalino adulto, apresentam aberração esférica positiva, ou seja, além de não compensarem a aberração esférica corneal (em média $+0.27 \mathrm{~mm}$ ), mantém a mesma indução adicional de aberração positiva do cristalino maduro. Entretanto, os pacientes, em geral, não se queixam, pois era a aberração óptica que eles, por muito tempo, já apresentavam devido à catarata. Porém, com implante de LIOs esféricas, são privados da oportunidade, teórica, de se melhorar ainda mais a visão, após a facectomia.

A solução proposta para compensar a aberração esférica é o implante da LIO asférica (aberração negativa), que corrigirá, ao menos parcialmente, a direção dos raios incidentes periféricos (aberração positiva) ${ }^{(13-37)}$.
Para avaliação do comprometimento visual pela catarata, assim como do benefício clínico das LIOs asféricas em relação às LIOs convencionais, o teste de Snellen, embora seja o instrumento mais utilizado para a avaliação da acuidade visual, apresenta limitações, pois mede a visão utilizando-se do contraste máximo (letras pretas em fundo branco). No mundo real a maioria dos objetos a serem visualizados não se encontra com tanto contraste, assim, a acuidade visual medida no consultório por meio deste teste não corresponde, necessariamente, a dificuldade de visão percebida pelo paciente no seu dia a dia. Deste modo, uma pessoa com catarata de componente nuclear predominante pode, por exemplo, enxergar 20/ 25 na tabela de Snellen e ter dificuldade para dirigir. Se o oftalmologista só considerar a visão medida com máximo contraste talvez não compreenda a queixa do paciente, provavelmente, em situações de menor contraste, como ao entardecer. Por outro lado, caso o paciente ou seu acompanhante se lembre de qual era a medida da visão no teste de Snellen antes da cirurgia, pode não se maravilhar com o resultado visual pós-operatório, que será,neste caso, provavelmente, de melhora de uma linha, mesmo que a dificuldade visual tenha sido corrigida.

Para se estimar mais precisamente a dificuldade visual pela catarata e para avaliar o desempenho visual com LIOs asféricas, o teste mais indicado é o de sensibilidade ao contraste, que mede a acuidade visual sob diferentes níveis de contraste. É por isso que a maioria dos estudos utiliza o teste de sensibilidade ao contraste (ETDRS) em condições mesópicas e fotópicas, além da avaliação objetiva da qualidade óptica por meio de exames de aberrometria ("frente de onda") (38).

A maioria dos estudos que compararam LIOs asféricas e esféricas não demonstraram diferenças em relação a melhor acuidade visual corrigida, medida pela tabela de Snellen ou pela do ETDRS, em condições fotópicas, exceto dois estudos ${ }^{(13,17)}$, que apresentaram resultados de melhor acuidade visual corrigida em pacientes com LIO Tecnis (AMO), se comparado aos pacientes com LIOs esféricas SA60AT (Alcon Labs) e SI40 (Alergan) ${ }^{(12-15,17,20-22,25,28,29,33,34,39)}$.

O resultado da maioria dos artigos nos leva a concluir que não há diferença entre LIOs asféricas e esféricas em relação a melhor acuidade visual, corrigida em condições de alta luminosidade, ou que, este tipo de teste não apresenta condições de detectar tal diferença.

Em condições mesópicas, as vantagens visuais das LIOs asféricas em relação às esféricas, quando avaliadas pelo teste do ETDRS, passam a ser mais evidentes. Nos trabalhos com a LIO Tecnis (AMO), apenas dois não encontraram diferenças, estatisticamente significa- 
tivas, quando comparadas a LIO Stabibag (Zeiss) ${ }^{(20)}$ e AR40e (AMO $)^{(20,22)}$. A maioria das pesquisas apresentou resultados superiores com a LIO asférica em freqüências espaciais que variaram de 1.5 a 30 ciclos por grau. Todos os estudos que avaliaram a LIO Acrysof IQ (Alcon labs) em condições mesópicas, demonstraram o melhor desempenho desta, quando comparada a diferentes LIOs esféricas ${ }^{(21,28,31,32,34,36)}$. Um estudo ${ }^{(28)}$, avaliou a LIO asférica SofPort (Bausch \& Lomb), e, demonstraram valores estatisticamente significativos, comparado às LIOs esféricas SN60AT (Alcon labs) e AR40e (AMO).

As lentes asféricas variam em relação a asfericiade, de forma que, as LIOs mais utilizadas, Tecnis (AMO), Acrysof IQ (Alcon labs) Akreos AO (Bausch \& Lomb), apresentam diferentes potenciais para compensação da aberração esférica da córnea. A aberração esférica corneal na média populacional é de $0.27 \mathrm{~mm}$ positivos; teoricamente, em condições de diâmetro pupilar de $6 \mathrm{~mm}$, a LIO Akreos AO (Bausch \& Lomb) apresenta $0.0 \mathrm{~mm}$ de aberração esférica residual, ou seja são livres de aberração; a LIO Tecnis (AMO) possui asfericidade de $-0.27 \mathrm{~mm}$, tentando compensar totalmente a aberração corneal considerando a média populacional e a LIO Acrysof IQ (Alcon labs) - $0.20 \mathrm{~mm}$, que deixa, se considerada a média populacional, um residual de aberração positiva de $0,07 \mathrm{~mm}$.

Estudo publicado recentemente ${ }^{(40)}$ que comparou duas LIOs asféricas, a Akreos AO (Baush \& Lomb) e a Tecnis (AMO), não demonstrou diferenças estatisticamente significativas, na acuidade visual, tanto em condições fotópicas, quanto em mesópicas, porém, por meio do exame de aberrometria (frente de onda) confirmou valores residuais maiores de aberração esférica com a LIO Akreos AO (Baush \& Lomb), sugerindo, assim, que o exame da acuidade visual avalia o desempenho do Sistema Visual total (globo ocular e córtex cerebral, incluindo a neuroadaptação), enquanto que o exame de frente de onda avalia apenas a porção óptica do olho (Sistema Óptico), ou seja, a qualidade da imagem que chega na retina.

Em determinados casos, a aberração esférica positiva residual pode ser benéfica, pois embora possa diminuir a visão de longe no escuro, aumenta a profundidade de foco, facilitando a visão de perto. Artigo recente ${ }^{(27)}$ demonstrou que a LIO esférica SN60AT (Alcon labs) apresenta maior profundidade de foco comparado a LIO asférica Acrysof IQ (Alcon labs), analisando visão intermediaria e de perto, em pacientes corrigidos para longe.

Considera-se também que a tolerância ao "defocus", pequenos erros refracionais esféricos, parece ser maior em pacientes com LIOs esféricas e/ou com residual de aberração esférica positiva. Esse seria um beneficio teórico da LIO asférica livre de aberração, como a Akreos AO (Baush \& Lomb), que deixaria aberração residual. Estudo ${ }^{(4)}$ que comparou a profundidade de foco em pacientes com LIOs asféricas livres de aberração, Akreos AO (Baush \& Lomb) e LIOs asféricas com aberração negativa, Tecnis (AMO) que tendem a compensar totalmente a aberração esférica positiva da córnea, concluiu que, a profundidade de foco é maior no grupo com LIOs asféricas livres de aberração.

Atualmente se tem estudado as LIOs asféricas, com objetivo de se personalizar a cirurgia de catarata, obtendo-se valores de aberração esférica residuais finais em torno de $0.10 \mathrm{~mm}$, com possível melhora na qualidade óptica e acuidade visual ${ }^{(41-46)}$. Artigo recente ${ }^{(47)}$ sugere que para pacientes com aberração esférica corneana entre $-0.15 \mathrm{e}+0.15$ poderia ser implantada uma LIO asférica livre de aberração, como a Akreos AO (Baush \& Lomb), em pacientes com valores entre +0.16 e +0.33 Acrysof IQ (Alcon labs), e para pacientes com valores acima de +0.33 , a LIO Tecnis (AMO).

$\mathrm{Na}$ indicação de LIOs asféricas, alguns fatores individuais também devem ser considerados, como diâmetro pupilar e histórico de cirurgia refrativa à laser.

Embora consideremos que o sistema visual dispõe de um mecanismo compensatório de aberrações ópticas, que é a neuroadaptação, capaz de, em muitos casos, resolver o problema da aberração esférica, bem como de outras deficiências ópticas, por si só, existe a tendência para personalizar a cirurgia de catarata, a fim de se obter resultados visuais ainda melhores ${ }^{(43,48)}$. No futuro, assim como é feita a biometria para se determinar o valor dióptrico da LIO a ser implantada, a fim de se corrigir os erros refrativos esféricos (aberrações de baixa ordem) e, em certos casos, realizado a topografia para se estimar o astigmatismo corneal (aberração de baixa ordem), objetivando-se compensá-lo com incisões relaxantes limbares ou LIOs tóricas, talvez também possa ser realizado, rotineiramente, o exame de "frente de onda", para se estimar a aberração esférica da córnea, a fim que esta possa ser compensada com determinada LIO asférica.

\section{Abstract}

With aspherical intraocular lens (IOL), phakectomy could be more than removal of cataract, it also could means an improvement in optical quality of the eye. In this article, recently published studies on aspherical IOLs and exams to access optical quality were reviewed.

Keywords: Lenses, intraocular; Cataract; Visual acuity/physiology 


\section{RefERÊNCIAS}

1. Applegate RA, Thibos LN, Hilmantel G. Optics of aberroscopy and super vision. J Cataract Refract Surg. 2001;27(7):1093107.

2. Applegate RA. Limits to vision: can we do better than nature? J Refract Surg. 2000;16(5):S547-51.

3. Williams DR, Liang J, Miller DT. Adaptive optics for the human eye. Vision science and its applications 1996 OSA Technical Digest Series. (Optical Society of America. Washington, DC). 1996;13:145-7.

4. Liang J, Williams DR, Miller DT. Supernormal vision and highresolution retinal imaging through adaptive optics. J Opt Soc Am A Opt Image Sci Vis. 1997;14(11):2884-92.

5. Bille JF. Preoperative simulation of outcomes using adaptive optics. J Refract Surg. 2000;16(5):S608-10.

6. He JC, Gwiazda J, Thorn F, Held R. Wave-front aberrations in the anterior corneal surface and the whole eye. J Opt Soc Am A Opt Image Sci Vis. 2003; 20(7):1155-63.

7. Brunette I, Bueno JM, Parent M, Hamam H, Simonet P. Monochromatic aberrations as a function of age, from childhood to advanced age. Invest Ophthalmol Vis Sci. 2003;44(12):5438-46.

8. Smith G, Cox MJ, Calver R, Garner LF. The spherical aberration of the crystalline lens of the human eye. Vision Res. 2001;41(2):235-43

9. Holladay JT, Piers PA, Koranyi G, van der Mooren M, Norrby NE. A new intraocular lens design to reduce spherical aberration of pseudophakic eyes. J Refract Surg. 2002;18(6):68391.

10. Artal P, Berrio E, Guirao A, Piers P. Contribution of the cornea and internal surfaces to the change of ocular aberrations with age. J Opt Soc Am A Opt Image Sci Vis. 2002;19(1):137-43.

11. Wang L, Koch DD. Ocular higher-order aberrations in individuals screened for refractive surgery. J Cataract Refract Surg. 2003;29(10):1896-903

12. Packer M, Fine H, Hoffman RS, Piers PA. Prospective randomized trial of an anterior surface modified prolate intraocular lens. J Refract Surg. 2002;18(6):692-6.

13. Mester U, Dillinger P, Anterist N. Impact of a modified optic design on visual function: clinical comparative study. J Cataract Refract Surg. 2003;29(4):652-60. Comment in: J Cataract Refract Surg. 2003;29(4):627-8.

14. Kershner RM. Retinal image contrast and functional visual performance with aspheric, silicone, and acrylic intraocular lenses. Prospective evaluation. J Cataract Refract Surg. 2003;29(9):1684-94.

15. Packer M, Fine IH, Hoffman RS, Piers PA. Improved functional vision with a modified prolate intraocular lens. J Cataract Refract Surg. 2004;30(5):986-92. Comment in: J Cataract Refract Surg. 2004;30(11):2247-8; author reply 2248-50.

16. Bellucci R, Morselli S, Piers P. Comparison of wavefront aberrations and optical quality of eyes implanted with five different intraocular lenses. J Refract Surg. 2004;20(4):297-306.

17. Bellucci R, Scialdone A, Buratto L, Morselli S, Chierego C, Criscuoli A, et al. Visual acuity and contrast sensitivity comparison between Tecnis and AcrySof SA60AT intraocular lenses: A multicenter randomized study. J Cataract Refract Surg. 2005;31(4):712-7. Erratum in: J Cataract Refract Surg. 2005;31(10):1857.

18. Marcos S, Barbero S, Jiménez-Alfaro I. Optical quality and depth-of-field of eyes implanted with spherical and aspheric intraocular lenses. J Refract Surg. 2005;21(3):223-35.
19. Kasper T, Bühren J, Kohnen T. Intraindividual comparison of higher-order aberrations after implantation of aspherical and spherical intraocular lenses as a function of pupil diameter. $\mathbf{J}$ Cataract Refract Surg. 2006;32(1):78-84.

20. Muñoz G, Albarrán-Diego C, Montés-Micó R, RodríguezGalietero A, Alió JL. Spherical aberration and contrast sensitivity after cataract surgery with the Tecnis Z9000 intraocular lens. J Cataract Refract Surg. 2006;32(8):1320-7. Comment in: J Cataract Refract Surg. 2007;33(3):359-60; author reply 360-1.

21. Rocha KM, Soriano ES, Chalita MR, Yamada AC, Bottós K, Bottós J, et al. Wavefront analysis and contrast sensitivity of aspheric and spherical intraocular lenses: a randomized prospective study. Am J Ophthalmol. 2006;142(5):750-6.

22. Kasper T, Bühren J, Kohnen T. Visual performance of aspherical and spherical intraocular lenses: intraindividual comparison of visual acuity, contrast sensitivity, and higher-order aberrations. J Cataract Refract Surg. 2006;32(12):2022-9.

23. Padmanabhan P, Rao SK, Jayasree R, Chowdhry M, Roy J. Monochromatic aberrations in eyes with different intraocular lens optic designs. J Refract Surg. 2006;22(2):172-7.

24. Bellucci R, Morselli S, Pucci V. Spherical aberration and coma with an aspherical and a spherical intraocular lens in normal age-matched eyes. J Cataract Refract Surg. 2007;33(2):2039. Comment in: J Cataract Refract Surg. 2007;33(2):173-4.

25. Denoyer A, Le Lez ML, Majzoub S, Pisella PJ. Quality of vision after cataract surgery after Tecnis Z9000 intraocular lens implantation: effect of contrast sensitivity and wavefront aberration improvements on the quality of daily vision. J Cataract Refract Surg. 2007;33(2):210-6. Comment in: J Cataract Refract Surg. 2007;33(2):173-4.

26. Kurz S, Krummenauer F, Thieme H, Dick HB. Contrast sensitivity after implantation of a spherical versus an aspherical intraocular lens in biaxial microincision cataract surgery. J Cataract Refract Surg. 2007;33(3):393-400.

27. Rocha KM, Soriano ES, Chamon W, Chalita MR, Nosé W. Spherical aberration and depth of focus in eyes implanted with aspheric and spherical intraocular lenses: a prospective randomized study. Ophthalmology. 2007;114(11):2050-4. Comment in: Ophthalmology. 2008;115(8):1438-9; author reply $1439-40$. Ophthalmology. 2008;115(9):1641; author reply $1641-2$.

28. Caporossi A, Martone G, Casprini F, Rapisarda L. Prospective randomized study of clinical performance of 3 aspheric and 2 spherical intraocular lenses in 250 eyes. J Refract Surg. 2007;23(7):639-48.

29. Sandoval HP, Fernández de Castro LE, Vroman DT, Solomon KD. Comparison of visual outcomes, photopic contrast sensitivity, wavefront analysis, and patient satisfaction following cataract extraction and IOL implantation: aspheric vs spherical acrylic lenses. Eye. 2008;22(12):1469-75.

30. Awwad ST, Lehmann JD, McCulley JP, Bowman RW. A comparison of higher order aberrations in eyes implanted with AcrySof IQ SN60WF and AcrySof SN60AT intraocular lenses. Eur J Ophthalmol. 2007;17(3):320-6.

31. Pandita D, Raj SM, Vasavada VA, Vasavada VA, Kazi NS, Vasavada AR. Contrast sensitivity and glare disability after implantation of AcrySof IQ Natural aspherical intraocular lens: prospective randomized masked clinical trial. J Cataract Refract Surg. 2007;33(4):603-10.

32. Tzelikis PF, Akaishi L, Trindade FC, Boteon JE. Ocular aberrations and contrast sensitivity after cataract surgery with AcrySof IQ intraocular lens implantation Clinical Comparative Study. J Cataract Refract Surg. 2007;33(11):1918-24. 
33. Tzelikis PF, Akaishi L, Trindade FC, Boteon JE. Spherical aberration and contrast sensitivity in eyes implanted with aspheric and spherical intraocular lenses: a comparative study. Am J Ophthalmol. 2008;145(5):827-33.

34. Awwad ST, Warmerdam D, Bowman RW, Dwarakanathan S, Cavanagh HD, McCulley JP. Contrast sensitivity and higher order aberrations in eyes implanted with Acrysof IQ SN60WF and AcrySof SN60AT intraocular lenses. J Refract Surg. 2008;24(6):619-25.

35. Cadarso L, Iglesias A, Ollero A, Pita B, Montés-Micó R. Postoperative optical aberrations in eyes implanted with AcrySof spherical and aspheric intraocular lenses. J Refract Surg. 2008;24(8):811-6.

36. Mester U, Kaymak H. Comparison of the AcrySof IQ aspheric blue light filter and the Acrysof SA60AT intraocular lenses. J Refract Surg. 2008;24(8):817-25.

37. Atchison DA. Design of aspheric intraocular lenses. Ophthalmic Physiol Opt. 1991;11(2):137-46.

38. Ginsburg AP. Contrast sensitivity: determining the visual quality and function of cataract, intraocular lenses and refractive surgery. Curr Opin Ophthalmol. 2006;17(1):19-26.

39. Montés-Micó R, Ferrer-Blasco T, Cerviño A. Analysis of the possible benefits of aspheric intraocular lenses: review of the literature. J Cataract Refract Surg. 2009;35(1):172-81.

40. Johansson B, Sundelin S, Wikberg-Matsson A, Unsbo P, Behndig A. Visual and optical performance of the Akreos Adapt Advanced Optics and Tecnis Z9000 intraocular lenses: Swedish multicenter study. J Cataract Refract Surg. 2007;33(9):1565-72.
41. Grimson JM, Schallhorn SC, Kaupp SE. Contrast sensitivity: establishing normative data for use in screening prospective naval pilots. Aviat Space Environ Med. 2002;73(1):28-35.

42. Levy Y, Segal O, Avni I, Zadok D. Ocular higher-order aberrations in eyes with supernormal vision. Am J Ophthalmol. 2005;139(2):225-8.

43. Wang L, Koch DD. Custom optimization of intraocular lens asphericity. J Cataract Refract Surg .2007;33(10):1713-20.

44. Wang L, Dai E, Koch DD, Nathoo A. Optical aberrations of the human anterior cornea. J Cataract Refract Surg. 2003;29(8):1514-21.

45. Beiko GH, Haigis W, Steinmueller A. Distribution of corneal spherical aberration in a comprehensive ophthalmology practice and whether keratometry can predict aberration values. J Cataract Refract Surg. 2007;33(5):848-58.

46. Applegate RA, Marsack JD, Ramos R, Sarver EJ. Interaction between aberrations to improve or reduce visual performance. J Cataract Refract Surg. 2003;29(8):1487-95.

47. Beiko G. Aspheric IOLs: matching corneal and IOL wavefront. In: Chang DF, editor. Mastering refractive IOLs: the art and science. Thorofare, NJ: Slack; 2008. p. 278-81.

48. Packer M, Fine IH, Hoffman RS. Aspheric intraocular lens selection based on corneal wavefront. J Refract Surg. 2009;25(1):12-20. 